\title{
The insulin-like growth factor 2 (IGF2) mRNA-binding protein p62/IGF2BP2-2 as a promoter of NAFLD and HCC?
}

Non-alcoholic fatty liver disease (NAFLD) represents the most common hepatic manifestation of chronic liver diseases in developed countries. Since non-alcoholic steatohepatitis (NASH) is responsible for a large proportion of cryptogenic cirrhosis and cirrhosis represents the main risk factor for hepatocellular carcinoma (HCC), HCC is a severe complication of end-stage NAFLD. ${ }^{1}$

Recent evidence published in this journal showed the therapeutic potential of an inhibition of the chemokine (C-C motif) ligand 2 (CCL2)/monocyte chemoattractant protein-1 (MCP-1) in NASH. ${ }^{2}$ The study by Baeck et al elegantly demonstrated that the pharmacological administration of an RNA oligonucleotide against MCP-1 ameliorates murine steatosis and inflammation. Since mice deficient of the MCP-1 receptor also showed attenuated fibrosis, MCP-1 was suggested as a critical link in the axis steatosis-inflammationfibrosis. $^{2}$

Here, we report that animals with a liverspecific overexpression of the insulin-like growth factor 2 (IGF2) mRNA-binding protein p62/IMP2-2/IGF2BP2-2 exhibit distinctly elevated $\mathrm{Ccl} 2$ expression levels (figure 1A) when fed a methionine-cholinedeficient (MCD) diet, which models all hepatic stages of NAFLD. Accordingly, in addition to elevated inflammatory gene expression, $p 62$ transgenics had higher fat deposition (figure 1B) and an earlier onset and more pronounced manifestation of fibrosis than their wild-type littermates on MCD diet (figure 1B). These data further support a critical role for MCP-1 in NASH and $\mathrm{NASH}$-induced fibrosis as suggested previously. $^{2}$

The autoantigen p62 was originally isolated from a patient with HCC and was found to be overexpressed in HCC patients and in premalignant cirrhotic nodules. $^{3-5}$ We previously reported that p62 transgenic animals on regular chow develop a fatty liver. ${ }^{6}$ Our new findings suggest that the lipogenic action of p62 on the MCD diet is facilitated by the induction of the lipogenic transcription factor Srebp1c (figure 1C). Interestingly, sterol regulatory element binding protein (SREBP) has also been reported to play a critical role in HCC lipogenesis and correlates with a poor prognosis in HCC. ${ }^{1}$

In order to elucidate the molecular mechanism of fibrogenesis in $p 62$ 
A

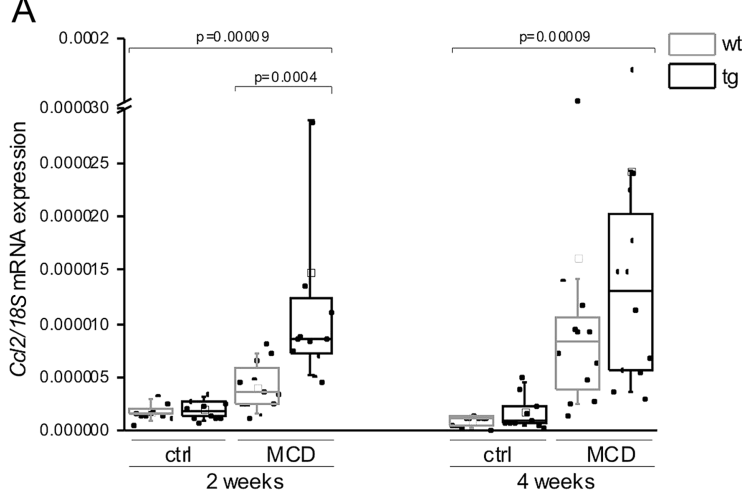

B $\quad$ w
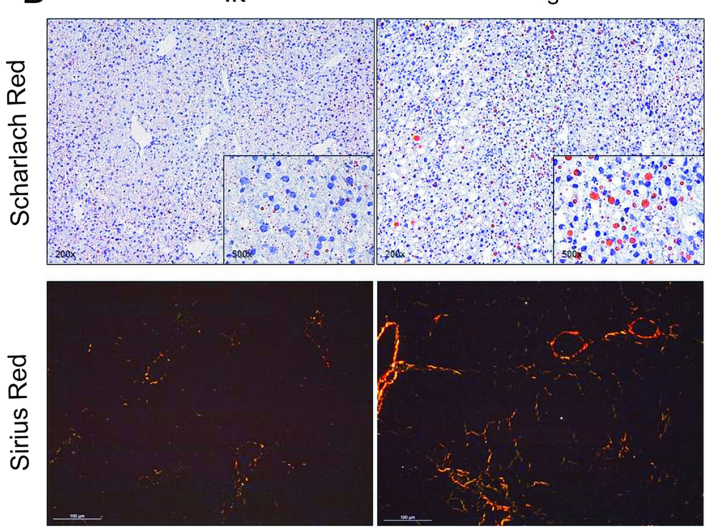

C
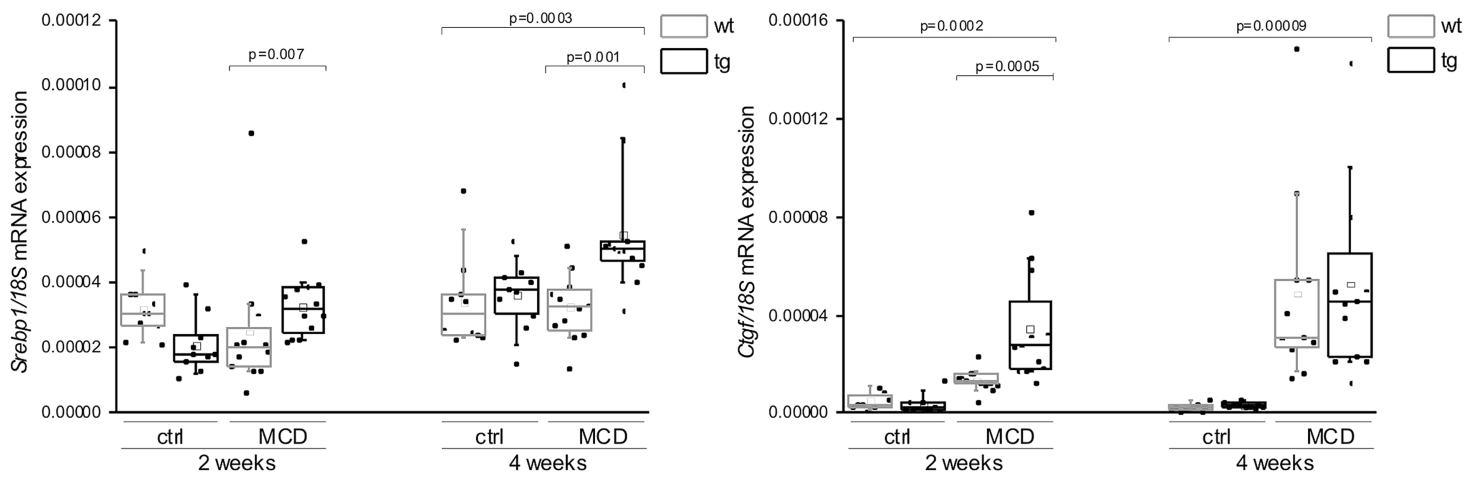

Figure 1 p62 amplifies steatosis, inflammation and fibrosis in murine methionine-choline-deficient (MCD)-induced non-alcoholic steatohepatitis. (A) Gene expression of Ccl2/Mcp1 normalised to $18 \mathrm{~S}$ of wild-type (wt) or p62 transgenic (tg) livers on control (ctrl) or MCD diet. (B) Lipid accumulation after 2 weeks on MCD diet is visualised by Scharlach Red stain and hepatic collagen deposition after 4 weeks on MCD diet is visualised by polarisation microscopy after Sirius Red staining. (C) Relative hepatic mRNA expression of Srebp1c normalised to 18S. (D) Relative mRNA expression of Ctgf normalised to 18S. Data in A, C and D are represented as individual values and box plots with median ( - ) and mean in a five-number summary $(n=9-12)$.

transgenic mice on the MCD diet, the expression levels of transforming growth factor $\beta(T g f b)$ and its downstream target gene connective tissue growth factor (Ctgf) were measured. Surprisingly, Tgfb levels were not elevated in animals fed the MCD diet (data not shown), whereas Ctgf expression was induced (figure 1D), suggesting a TGF- $\beta$-independent production of collagen. Liu et $a l^{7}$ demonstrated that the cytokine interleukin (IL)-13 induces Ctgf irrespective of TGF- $\beta$. Interestingly, serum IL-13 levels were increased in $p 62$ transgenic mice $(p=0.002)$, suggesting a p62-induced, IL-13-dependent Ctgf expression resulting in liver fibrosis. Similarly, in human NASH patients, serum IL-13 levels are increased and the inhibition of the IL-13 receptor in a rat model of NASH led to a reduction of fibrosis. ${ }^{8}$

These findings on p62 in the MCD mouse model strongly support a pathophysiological role of the tumour-associated autoantigen p62 in all stages of NAFLD, probably involving the induction of MCP-1. In fact, recent data also suggested a potential role for MCP-1 in HCC. ${ }^{9}$
We suggest that $\mathrm{p} 62$ might also drive the progression from NAFLD towards HCC. Interestingly, p62 induces or amplifies several of the most important mechanistic links between NAFLD and HCC as highlighted by Stickel and Hellerbrand. ${ }^{1}$ These comprise, for example, a downregulation of the tumour suppressor phosphatase and tensin homolog ${ }^{16}$ or the activation of pro-oncogenic signalling pathways, such as extracellular-signal regulated kinase. ${ }^{14}$ The induction of the lipogenic Srebp transcription factor as reported above might also be of special interest. ${ }^{1}$

Having in mind that $\mathrm{p} 62$ expression correlates with poor outcome in HCC, ${ }^{4}$ our data suggest that p62 potentially represents a critical pathophysiological factor linking NAFLD to hepatocarcinogenesis.

Yvette Simon, ${ }^{1}$ Sonja M Kessler, ${ }^{1,2}$ Rainer M Bohle, ${ }^{3}$ Johannes Haybaeck, ${ }^{2}$ Alexandra K Kiemer ${ }^{1}$

${ }^{1}$ Department of Pharmacy, Saarland University, Pharmaceutical Biology, Saarbrücken, Saarland, Germany

${ }^{2}$ Institute of Pathology, Medical University of Graz, Graz, Austria
${ }^{3}$ Department of Pathology, Saarland University, Homburg/Saar, Germany

Correspondence to Prof. Alexandra K Kiemer, Department of Pharmacy, Saarland University, Pharmaceutical Biology, P.O. box 1511 50, Saarbrücken 66041, Germany; pharm.bio.kiemer@mx. uni-saarland.de

Acknowledgements We thank Eva Dilly for the technical assistance in all animal matters. We thank Christina Guth for the possibility to use the polarisation microscope at the Leibniz Institute for New Materials (INM).

Collaborators Eva Dilly; Christina Guth.

Contributors YS, SMK and AKK designed

experiments, analysed data and wrote the manuscript. AKK initiated and directed the study. SMK, RMB and JH scored the histological slides.

Funding The project was funded in part by an EASL Sheila Sherlock and a Bank Austria Visiting Scientists Program Fellowship to SMK.

Competing interests None.

Provenance and peer review Not commissioned; internally peer reviewed.

Data sharing statement All authors had full access to all of the data (including statistical reports and tables) in the study and can take responsibility for the integrity of the data and the accuracy of the data analysis. 
Open Access This is an Open Access article distributed in accordance with the Creative Commons Attribution Non Commercial (CC BY-NC 3.0) license, which permits others to distribute, remix, adapt, build upon this work non-commercially, and license their derivative works on different terms, provided the original work is properly cited and the use is non-commercial. See: http:// creativecommons.org/licenses/by-nc/3.0/

To cite Simon Y, Kessler SM, Bohle R M, et al. Gut 2014;63:861-863.

Received 23 July 2013

Revised 30 August 2013

Accepted 4 September 2013

Published Online First 30 October 2013

Gut 2014;63:861-863.

doi:10.1136/gutjnl-2013-305736

\section{REFERENCES}

1 Stickel F, Hellerbrand C. Non-alcoholic fatty liver disease as a risk factor for hepatocellular carcinoma: mechanisms and implications. Gut 2010;59:1303-7.

2 Baeck C, Wehr A, Karlmark KR, et al. Pharmacological inhibition of the chemokine CCL2 (MCP-1) diminishes liver macrophage infiltration and steatohepatitis in chronic hepatic injury. Gut 2012;2:1-12.

3 Lu M, Nakamura RM, Dent ED, et al. Aberrant expression of fetal RNA-binding protein p62 in liver cancer and liver cirrhosis. Am J Pathol 2001;159:945-53.

4 Kessler SM, Pokorny J, Zimmer V, et al. IGF2 mRNA binding protein p62/IMP2-2 in hepatocellular carcinoma: antiapoptotic action is independent of IGF2/PI3 K signaling. AmJ Physiol Gastrointest Liver Physiol 2013;4:G328-36.

5 Zhang J-Y, Chan EKL, Peng X-X, et al. A novel cytoplasmic protein with RNA-binding motifs is an autoantigen in human hepatocellular carcinoma. J Exp Med 1999;189:1101-10.

6 Tybl E, Shi F-D, Kessler SM, et al. Overexpression of the IGF2-mRNA binding protein p62 in transgenic mice induces a steatotic phenotype. J Hepatol 2011;54:994-1001.

7 Liu Y, Meyer C, Müller A, et al. IL-13 Induces connective tissue growth factor in rat hepatic stellate cells via TGF-beta-independent Smad signaling. J Immunol 2011;187:2814-23.

8 Shimamura T, Fujisawa T, Husain SR, et al. Novel role of IL-13 in fibrosis induced by nonalcoholic steatohepatitis and its amelioration by IL-13R-directed cytotoxin in a rat model. $J$ Immunol 2008; 181:4656-65.

9 Wang W-W, Ang SF, Kumar R, et al. Identification of serum monocyte chemoattractant protein-1 and prolactin as potential tumor markers in hepatocellular carcinoma. PLoS One 2013;8:e68904. 